\title{
Optimal Load Factor for Approximate Nearest Neighbor Search under Exact Euclidean Locality Sensitive Hashing
}

\author{
Ruben Buaba \\ Autonomous Control and \\ Information Technology Center, \\ Department of Electrical and \\ Computer Engineering \\ North Carolina Agricultural and \\ Technical State University \\ Greensboro, NC 27411
}

\author{
Abdollah Homaifar \\ Autonomous Control and \\ Information Technology Center, \\ Department of Electrical and \\ Computer Engineering \\ North Carolina Agricultural and \\ Technical State University \\ Greensboro, NC
}

\author{
Eric Kihn \\ NOAA /NGDC \\ 325 Broadway Boulder,CO \\ 80305
}

\begin{abstract}
Locality Sensitive Hashing (LSH) is an index-based data structure that allows spatial item retrieval over a large dataset. The performance measure, $\rho$, has significant effect on the computational complexity and memory space requirement to create and store items in this data structure respectively. The minimization of $\rho$ at a specific approximation factor $c$, is dependent on the load factor, $\alpha$. Over the years, $\alpha=4$ has been used by researchers. In this paper, we demonstratethat the choice of $\alpha=4$ does not guarantee low computational complexity and low memory space of the data structure under the LSH scheme. To guarantee low computational complexity and low memory space, we propose $\alpha=5$. Experiments on the Defense Meteorological Satellite Program imagery datasethave shown that $\alpha=5$ saves more than $75 \%$ on memory space; cuts the computational complexity by more than $70 \%$ andanswers query two times faster on the average compared to that of $\alpha=4$.
\end{abstract}

\section{General Terms}

Nearest Neighbor, Search Algorithm, Locality Sensitive Hashing

\section{Keywords}

Approximate Nearest Neighbor, Exact Nearest Neighbor, ApproximationFactor, Performance Measure,Optimal Load Factor

\section{INTRODUCTION}

\subsection{Nearest Neighbor Search}

A nearest neighbor (NN) search is composed as follows: given a set $\mathcal{P}$ of $n$ data pointsin a metric space, $X$, the task is to preprocess these points sothat, given any query point $q \in \mathcal{P}$ the data point nearest to $q$ can be reported quickly. This is also referred to as the closest-pointproblem or the post office problem[1]. Even though linear search (LS) algorithm guarantees the retrieval of the exact nearest data point to a given query point correctly, it becomes computationally exhaustive and queryruntime complexity can be exponential when dealing with a large datasetwithhigh dimensionality. The reason being,LS literally iterates through the entire dataset and computes some metric distance between each data point and the query point and then returns the data point closest to the query point.
In practice, the $\mathrm{NN}$ search problem involves a collection of large number of items characterized by high dimensionality. In reality, the dataset for most applications is dynamic. In other words, the dataset is updated as when new data is collected. Consequently, similarity search algorithm should scale to produce an output within a reasonable timespan irrespective of the growth of the dataset. Thus, building a data structure that can be used to index and store these items in such a manner that given any query item, the search algorithm is able to find the most similar itemin sublinearquery runtime is vital. This problem is of major importance including but not limited to image and video database retrieval, data compression, information retrieval, database and data mining, pattern recognition, statistics and data analysis[2,3].

Over the years, intensive research has been done either to use trees, K-means clustering/classification or hashes to develop a space-partitioned data structure that would have a sublinearquery runtime[4-8]. For uniformly distributed data points, expected query runtime is achievable by algorithms that decompose the search space into regular grids $[9,10]$. In [11], the authors generalized these results and reported that $O(n)$ space and $O(\log n)$ query time are possible using kdtrees. However, even these methods suffer as dimension (i.e. d) of the data points increases because the constant factors hidden in the asymptotic query runtime of the kd- trees grows as fast as $2^{d}$. In [12], the author experimentally measured the query runtime of kd-trees and observed that it increases quite rapidly with the dimension. Also in[13], it is shown that if $n$ is not substantially larger than $2^{d}$ (which arises in some realworld applications), boundary effects decrease this exponential dimensional dependency. Thus, perfect solution would be to preprocess the points in $O(n \log n)$ time, into a data structure that requires $O(n)$ space so that queries can be answered in $O(\log n)$ time. For one-dimensional data points, sorting the points, and then using binary search to answer queries achieves this goal. For two-dimensional data points, it is possible to compute the Voronoi diagram for the data points and then use any fast planar point location algorithm to locate the cell containing the query point [14-16]. However, for higher dimensional data points, the worst-case complexity of the Voronoi diagram grows as quickly as $O\left(n^{[d / 2]}\right) . \operatorname{In[17]}$, the authors provided higher-dimensional solutions with sublinear worst-case performance. In [18], it is reported that for some arbitrary constant $\delta>0$, queries could be answered 
in $O(\log n)$ time with $O\left(n^{[d / 2\rceil+\delta}\right)$ space with hidden constant factors that are exponential in $d$. In [19], the authors generalized this by providing a trade-off between space and query runtime. Later in[20], it is reported that exponential factors in query runtime could be eliminated with an algorithm having $O\left(d^{5} \log n\right)$ query time and $O\left(n^{d+\delta}\right)$ space.

Unfortunately, it is shown both theoretically and empiricallythat these solutions provide little or no improvement over the LS algorithm for highly dimensional large dataset[21, 22]. Consequently, several researchers have become proponents of the use of approximation similarity search algorithms[23-27]. The fundamental principle being, in practice, approximate nearest neighbor is almost as good as the exact nearest neighbor in most cases. Since a distance measure is what is often used for similarity estimation, a small difference in the distance should not adversely influence the similarity estimation unless the nearest neighbor problem itself is unstable[28, 29]. This notion of approximation forms the basis of a novel similarity search algorithm known as the Locality Sensitive Hashing (LSH) which was first introduced in[25]. This technique drastically reduces the query runtime at the expense of a small probability of failure to find the absolute closest match. The concept of the approximate nearest neighbor (ANN) in some $l_{s}$ norm space is formulated as follows: suppose $q$ is a query whose exact NN is $q^{*}$. For a given $c>1, \boldsymbol{p}$ is said to be a $c-N N$ of $\boldsymbol{q}$ if $\|\boldsymbol{p}-\boldsymbol{q}\|_{s} \leq$ $c\left\|\boldsymbol{p}-\boldsymbol{q}^{*}\right\|_{s}$.

The performance of LSH depends on the minimization ofthe performance measure, $\rho$ that is associated with an optimal load factor $\alpha$.This guides the memory requirement, the computational complexity and the query runtime of the data structure under the LSH scheme. Over the years, many researchers haveused $\alpha=4[25,21,30,31]$. In this paper, it is demonstrated that the choice of $\alpha=4$ does not guarantee low memory requirement and low computational complexity of the data structure under the LSH scheme. In addition, the query runtime is slow. We show that under the LSH scheme, a load factor of $\alpha=5$ guarantees a lower memory requirement, lowercomputational complexity and faster query runtime compared to the conventional choiceof $\alpha=4$.

The remainder of this paper is organized as follows: Section 1.2 formulates the problem; section 2 offers the background of LSH in general; section 3 explains theorems and parametric constraintsof our proposed technique; section 4 talks about the complexity of the LSH; section 5 offers an empirical implementation of LSH on real data; and section 6draws the conclusions and proposes a future improvement.

\subsection{Notations and Problem Definition}

Unless otherwise stated the following parameter notations are used throughout this paper. $\mathcal{P}$ is a set of $n$ data points in $d$ dimensional space $\left(\Re^{d}\right)$. A query data point and any other point are denoted $\boldsymbol{q}$ and $\boldsymbol{p}$ respectively such that $\boldsymbol{p} \neq \boldsymbol{q} \in \mathcal{P}$. A sphere of radius $R$ centered at $\boldsymbol{q}$ is denoted by $\beta(\boldsymbol{q}, R)$. For any $\varepsilon>0, c=1+\varepsilon$ is the approximation factor such that $c R>R$. The $l_{2}$ norm of $\boldsymbol{p}$ is denotedby $\|p\|_{2}$. The expected number of data points per bucket (i.e. load factor) is denoted by $\alpha$. Our goal is to build a data structure for $\mathcal{P}$ under the LSH scheme by choosing an optimal value for $\alpha$ that guarantees lower memory, lowercomputational complexityand faster query runtime than that of the existing load factor, $\alpha=4$.This data structure is to solve the $(R, c)$-nearest neighbor problem in the $l_{2}$ norm space defined as follows: if $\exists \boldsymbol{q}^{*}:\left\|\boldsymbol{q}-\boldsymbol{q}^{*}\right\|_{2} \leq$
$R$ then in sublinearquery runtime, report any point $\boldsymbol{p}$ : $\| \boldsymbol{q}-$ $\boldsymbol{p}_{\|_{2}} \leq c R$ if it such a point exists.

\section{LSH BACKGROUND}

LSH is an index-based data structure that allows spatial item retrieval over a large database. The basic idea underlying the operation and the effectiveness of the LSH is that if two data points $\boldsymbol{p}, \boldsymbol{q} \in \mathfrak{R}^{d}$ (i.e. $d \in Z^{+}$) are close, then after a scalar projection of these points onto a hyper-plane, the two points should remain close to each other. On the other hand, if the points are far apart, they should remain far apart from each other after a scalar projection onto that same hyper-plane. This assertion is true in most cases[25, 32].However, with small failure probability, $\delta$, some points that are far apart might become closer after projection onto a lower dimension. For a dynamic dataset (i.e. dataset grows from time to time), the major advantage of LSH over tree-data structures is its ability to support deletion and insertion [30] operations. Suppose the real number line is "chopped" into slots (i.e. bucket)numbered $0,1, \ldots, m-1$ to form a table. If integer values are assigned to the data points based on which slots they project to, then intuitively, making several projections certainly increases the probability, $P_{l}$ of nearby points projecting to the same slot and decrease the probability, $P_{2}$ of far points from projecting to same slot. To make further guarantee this, several families of hash functions are used to perform the scalar projections. To achieve this goal, the functions must be locality sensitive and universal[33].

Definition \#1: A family of hash functions, $\mathcal{H}=\left\{\boldsymbol{h}_{\boldsymbol{i j}}: \mathcal{P} \rightarrow U\right\}$ each $\boldsymbol{h}_{\boldsymbol{i} j}$ mapping one point from domain $\mathcal{P}$ to domain $U$, is said to be $P_{1}>P_{2}, c R>R$ locality-sensitive if for any $\boldsymbol{p}, \boldsymbol{q} \in \mathcal{P}$,

- $\quad$ if $\boldsymbol{p} \in \beta(\boldsymbol{q}, R)$ then $\operatorname{Pr}^{[}\left[h_{i j}(\boldsymbol{p})=h_{i j}(\boldsymbol{q})\right] \geq P_{1}\left({ }^{\prime} h i g h^{\prime}\right)$

- $\quad$ if $\boldsymbol{p} \notin \beta(\boldsymbol{q}, c R)$ then $\left.\operatorname{Pr}^{2} h_{i j}(\boldsymbol{p})=h_{i j}(\boldsymbol{q})\right] \leq P_{2}\left({ }^{\prime} l o w^{\prime}\right)$

Definition \#2: Suppose $\mathcal{P}$ is a universe of keys, and $\mathcal{H}$ is a family of a finite collection of hash functions, each mapping Uto $0,1, \ldots, m-1, \mathcal{H}$ is said to be universal if $\forall \boldsymbol{p}, \boldsymbol{q} \in$ $\mathcal{P}$ and $p \neq q$, then $\operatorname{Pr}\left[h_{i j} \in \mathcal{H}: h_{i j}(\boldsymbol{p})=h_{i j}(\boldsymbol{q})\right]=\mathcal{H} / \mathrm{m}$.

A well-developed hash function tries to amplify the gap between the two probabilities, $P_{1}$ and $P_{2}$. To guarantee this amplification $P_{1} / P_{2}, k$ hash functions are chosen identically and independently from $\mathcal{H}$. To further amplify the gap between $P_{1}$ and $P_{2}, L$ tables are created. Whenever two or more items hash into the same bucket on any table, collision, is said to have occurred and this is sometimes resolved using double hashing, linked-list, or chaining. Normally, the number of buckets is large. As a result, it is only the nonempty buckets that are retained after all the data points in Pare projected into the buckets on the various tables. Creation of hash tables is normally fast and simple if the objects in the dataset are binary strings, i.e. $\{01\}^{d}[21,25]$ the biggest drawback of LSH. In spite of this limitation, LSH algorithm has been used in a number of applications involving non-binary data set[34-40]. To handle non-binary dataset, the algorithm has to be extended to the $l_{2}$ norm, by embedding $l_{2}$ space into $l_{l}$ space, and then $l_{l}$ space into the binary Hamming space. This however increases the complexity of the search algorithm but the advantages achieved outweigh this drawback.

Once the hash table is created and stored, for any query point $\boldsymbol{q} \in \mathcal{P}$, the $(R, c)$-NNs can be found by hashing $\boldsymbol{q}$ and retrieving data points stored in the buckets $h_{1}(\boldsymbol{p}), h_{2}(\boldsymbol{p}), \ldots, h_{L}(\boldsymbol{p})$ in which the query point hashes into Therefore, when the load factor, $\alpha$ varies, there is a trade-off 
between a larger table with a smaller final linear search or a more compact table with more data points to consider in the final search. The $(R, c)$-NNs search is terminated after finding the first $2 L$ data points (including duplicates) closet to $\boldsymbol{q}[30]$. The parameters $k$ and $L$ are chosen such that the following two conditions hold with constant probabilities:

- Condition \#1: if $\boldsymbol{q}^{*} \in \beta(\boldsymbol{q}, R)$, then $h_{j}\left(\boldsymbol{q}^{*}\right)=h_{j}(\boldsymbol{q})$ for some $j=1,2, \ldots, L$

- Condition \#2: the expected number of collision of $\boldsymbol{q}$ with any point $\boldsymbol{p} \in \mathcal{P}$ such that $\boldsymbol{p} \in \beta(\boldsymbol{q}, c R)$ is less than $2 L$

\section{THEORY AND CONSTRAINTS}

\subsection{S-stable Distribution}

Stable distributions are defined as limits of normalized sums of independent identically distributed variables [41]. A distribution $D$ is said to be $s$-stable if there exists $s \geq 0$, such that for any $N$ real numbers $u_{1}, u_{2}, \ldots, u_{N}$ and independent identically distributed random variables, $X_{1}, X_{2}, \ldots, X_{N}$ with distribution $D$, the random variable, $\sum_{i=1}^{N} u_{i} X_{i}$ has the same distribution as the variable $\left(\sum_{i=1}^{N}\left|u_{i}\right|^{s}\right)^{1 / s} X$, where $X$ is a random variable with distribution $D$. For $s \in[0,2]$, there exist stable distributions[41]. However, the focus is shifted to the case, $s=2$ since the similarity measure which is normally used is the $l_{2}$ norm (the Euclidean space). Under this, the normal Gaussian distribution denoted, $\mathcal{N}(0,1)$ with a probability distribution function, $f(x)=\frac{1}{\sqrt{2 \pi}} e^{-x^{2} / 2}$ is 2 stable. Stable distributions have applications in many fields [34]. In computer science, stable distributions are used for "sketching" high dimensional vectors[25].

Suppose a random vector $\boldsymbol{h}_{\boldsymbol{i j}} \in \mathfrak{R}^{d}$ is chosen from the standard Gaussian distribution, $\boldsymbol{h}_{\boldsymbol{i} \boldsymbol{j}} \sim \mathcal{N}(0,1)$ and $\boldsymbol{p}$ is any vector such that $\boldsymbol{p} \in \mathfrak{R}^{d} \rightarrow \boldsymbol{p}=\left[p_{1}, p_{2}, \ldots, p_{d}\right]$. Then the scalar dot product $\boldsymbol{h}_{\boldsymbol{i} \boldsymbol{j}} \bullet \boldsymbol{p}$ is a random variable which tends to be distributed as $\|\boldsymbol{p}\|_{2} \boldsymbol{h}_{\boldsymbol{i} \boldsymbol{j}}$, where $\boldsymbol{h}_{\boldsymbol{i j}}$ is a random variable with 2-stable distribution and $\|p\|_{2}$ is the $l_{2}$ norm of vector $p$ given as $\|p\|_{2}=\left(\sum_{z=1}^{d} p_{z}^{2}\right)^{1 / 2}$. In other words, the difference, $\boldsymbol{h}_{\boldsymbol{i} \boldsymbol{j}} \bullet$ $\boldsymbol{p}-\boldsymbol{h}_{\boldsymbol{i j}} \bullet \boldsymbol{q}$ tends to be distributed as $\|\boldsymbol{q}-\boldsymbol{p}\|_{2} \boldsymbol{h}_{\boldsymbol{i j}}$. Small collections of such dot products corresponding to different $\boldsymbol{h}_{i j}$ can be used to estimate $\|\boldsymbol{q}-\boldsymbol{p}\|_{2}$, the $l_{2}$ norm between the two data points $\boldsymbol{p}, \boldsymbol{q} \in \mathcal{P}$. Hence the data structure under the LSH scheme is said to be $l_{2}$-embedding and the $l_{2}$ norm forms the similarity metric for finding the $\mathrm{NN}$ to a given query data point.

\subsection{Evaluation of $P 1$ and $P 2$}

Suppose $c=\|\boldsymbol{q}-\boldsymbol{p}\|_{2}$ and $b_{i j}$ is an offset drawn uniformly from $[0, \alpha]$ at random. Also assume that $h_{j}$ is a family of $k$ hash functions drawn randomly and independently from $\mathcal{N}(0,1)$ such that, $h_{j}=\left[\boldsymbol{h}_{\mathbf{1}}, \boldsymbol{h}_{\mathbf{2}}, \ldots, \boldsymbol{h}_{\boldsymbol{k} j}\right]$. The hash value of $\boldsymbol{p}$ can be computed as, $h_{j}(\boldsymbol{p})=\left[\left\lfloor\frac{\boldsymbol{h}_{\mathbf{1}} \bullet \boldsymbol{p}+b_{1 j}}{\alpha}\right\rfloor,\left\lfloor\frac{\boldsymbol{h}_{\mathbf{2 j}} \boldsymbol{\bullet} \boldsymbol{p}+b_{2 j}}{\alpha}\right\rfloor, \ldots,\left\lfloor\frac{\boldsymbol{h}_{\boldsymbol{k}} \boldsymbol{\bullet} \boldsymbol{p}+b_{k j}}{\alpha}\right\rfloor\right]$.From the 2-stable distribution, $\boldsymbol{h}_{\boldsymbol{i} \boldsymbol{j}} \bullet \boldsymbol{p}-\boldsymbol{h}_{\boldsymbol{i} \boldsymbol{j}} \bullet \boldsymbol{q}$ for any $\mathrm{i}^{\text {th }}$ hash function such $\boldsymbol{h}_{\boldsymbol{i} \boldsymbol{j}} \in h_{j}$ has the same distribution asc $\boldsymbol{h}_{\boldsymbol{i} \boldsymbol{j}}$. The probability that $\boldsymbol{p}$ and $\boldsymbol{q}$ collides is given by Equation (1). The $F(\bullet)$ in Equation(1) represents the probability density function of the absolute value of the Gaussian distribution.

$$
\operatorname{Pr}(c)=\operatorname{Pr}\left[h_{j}(\boldsymbol{p})=h_{j}(\boldsymbol{q})\right]=\int_{0}^{\alpha} \frac{1}{c} F\left(\frac{t}{c}\right)\left(1-\frac{t}{\alpha}\right) d t(\mathbf{1})
$$

It should be noted that for a given $\alpha$, the probability of collision decreases monotonically with $c$. This means that the probability of collision is high if $\|\boldsymbol{p}-\boldsymbol{q}\|_{2}$ is small and low if $\|\boldsymbol{p}-\boldsymbol{q}\|_{2}$ is large. Thus, as per Definition\#1, for this to be $P_{1}>P_{2}, c R>R$ sensitive $P_{1}=\operatorname{Pr}(c=1)$ and $P_{2}=\operatorname{Pr}(c>$ 1). Solving these yieldEquations (2) and (3) with $F_{c d f}(\bullet)$ being the cumulative distribution function of the Gaussian random variable.

$$
P_{1}=1-2 F_{c d f}(-\alpha)-\frac{2}{\sqrt{2 \pi} \alpha}\left(1-e^{\frac{-\alpha^{2}}{2}}\right)
$$

$$
P_{2}=1-2 F_{c d f}(-\alpha / c)-\frac{2}{\sqrt{2 \pi} \alpha / c}\left(1-e^{-\alpha^{2} / 2 c^{2}}\right)(\mathbf{3})
$$

These prior probabilities influence the performance measure, $\rho$ as shown in Equation (4).

$$
\rho=\ln P_{1} / \ln P_{2}
$$

\subsection{Computing the performance measure, $\rho$}

The two prior probabilities $P_{l}$ and $P_{2}$ are needed to compute $\rho$. From Equation(2), once $\alpha$ is known $P_{l}$ can be computed. To compute $P_{2}$ from Equation(3), both $\alpha$ and $c$ must be known. The $\rho$ directly affects the memory space required to store items in the hash table. As a result, the goal is to find $\alpha$ that minimizes $\rho$ at a specific $c$. In other words, the aim is to solve Equation(5).

$$
\min _{\forall \alpha \in Z^{+}}\left(\ln P_{1} / \ln P_{2}\right)
$$

There exists no closed-form solution for Equation(5). In [25], the authors provided an approximate solution as $\rho(c) \approx 1 / c$. A minimization tool such as Matlab could be used to find the optimal value for $\rho$. Later in[30], the authors conducted a minimization experiment in Matlab to solve Equation(5). The experiment was conducted for $c=\left(\begin{array}{ll}1 & 10\end{array}\right]$ in increment of 0.05 . For each value of $c$, the minimum value of $\rho(c)$ is computed over the range of $\alpha$. They concluded that their approach gave a minimum value of $\rho$ slightly below the approximate solution by[25]. Furthermore, they observed that $\rho$ is not very sensitive to $\alpha$ beyond a certain point; and as long $\alpha$ is chosen "sufficiently" away from 0 , the value of $\rho$ would be close to optimal. They added that, if $\alpha$ is too large, both $P_{1}$ and $P_{2}$ approach unity and this increases memory space and the query runtime.

\subsection{Proposed Optimal Load Factor}

Over the years, researchers use $\alpha=4$ proposed by [30]as the optimal load factor. This however, raises two major concerns. First, does the choice of $\alpha=4$ guarantee low computational complexityand low memory space to create and store a data structure for $n$ data points under the LSH scheme? Second, assuming $\alpha=4$ is indeed the optimal load factor, what is the 'best' optimal performance measure, $\rho$ to choose if $\alpha=4$ gives multiple distinct values for $\rho$ at different values of $c$ ? We address these concerns by repeating the same experiment by[30] after which a multi-objective optimization is used to find the actual optimal $\alpha$ and $\rho$.

In our experiment over the same specified range for $c$, there is a statistical frequency count of the number of times a specific optimal load factor, $\alpha_{\text {opt }}$ minimizes Equation(5) at different values of $c$ to give different optimal values of $\rho$ denoted

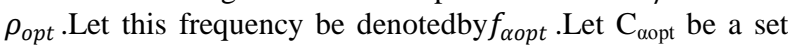
of the distinct values of $c$ for that $\alpha_{\text {opt }}$ such that $f_{\text {oopt }}>$ 1.Since the increment of $c$ is uniform, the $\alpha_{\text {opt }}$ withthe 
highest $f_{\text {copt }}$ has the widest span of $c$ in its corresponding set, $\mathrm{C}_{\text {aopt }}$. This means that the choice of $\operatorname{such} \alpha_{\text {opt }}$ would support a wider range of approximation factors. As per Definition \#1, $P_{l}$ must be 'high' and $P_{2}$ must be 'low'. Thus, the final choice of a specific $\alpha_{\text {opt }}$ depends not only on it having the highest $f_{\text {copt }}$ but also on the corresponding $c$ that maximizes $P_{l}$, and minimizes both $P_{2}$ and $\rho_{\text {opt }}$. The problem then becomes a multi-objective optimization. Let $P_{\text {lopt }}$ and $P_{2 o p t}$ be the optimal values of $P_{1}$ and $P_{2}$ respectively. Also let $\rho_{\text {aopt }}$ be the optimal value of $\rho_{\text {opt }}$. Suppose $\alpha_{\text {optMax }}$ is the optimal load factor with the highest frequency. Then, $P_{\text {lopt }}$ and $\rho_{\text {oopt }}$ can be obtained using Equations (6) and (7) respectively.

$$
\begin{aligned}
& P_{1 \text { opt }}=1-2 F_{c d f}\left(\alpha_{\text {optMax }}\right)-\frac{2}{\sqrt{2 \pi} \alpha_{\text {optMax }}}(1- \\
& e^{\left.\frac{-\alpha_{\text {optMax }}^{2}}{2}\right)}(6) \\
& \rho_{\alpha o p t} \min _{\forall \alpha \in C_{\alpha o p t}}\left\{\ln P_{1 o p t} /\left.\ln P_{2}(c)\right|_{\alpha=\alpha_{o p t M a x}}\right\}
\end{aligned}
$$

Suppose $\rho_{\text {oopt }}$ is obtained at $c=c_{\text {opt }} \in C_{\text {oopt }}$. Then, $P_{2 o p t}$ can be computed either using Equation(8) or (9).

$P_{2 o p t}=1-2 F_{c d f}\left(-\frac{\alpha_{o p t}}{c_{o p t}}\right)-\frac{2}{\sqrt{2 \pi} \frac{\alpha_{o p t}}{c_{o p t}}}\left(1-e^{-\alpha^{2} / 2 c_{o p t}^{2}}\right)$

(8)

$$
P_{2 o p t}=e^{\ln P_{1 o p t} / \rho_{\text {copt }}}
$$

Figure1 shows the optimal load factor, $\rho_{\text {opt }}$ from the minimization of $\rho(c)$ in Equation (5) and its approximate solution, $1 / c$ provided in[25]. In Figure 1, it is observed that the $\rho_{\text {opt }}$ curve lies slightly below that of $1 / c$.

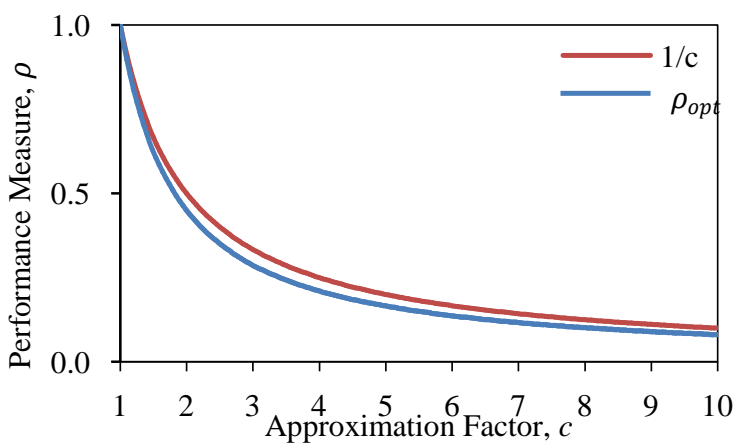

Figure1:The optimal performance parameter $\left(\rho_{\text {opt }}\right)$ and its corresponding approximate solution $(1 / \mathrm{c})$

Figure2 shows the frequency distributions of $\alpha_{o p t}$ for the minimization of $\rho$ inEquation(5). In Figure 2, it is observed that at $\alpha_{\text {opt }}=4$ and $\alpha_{\text {opt }}=5$ tied at the same highest frequency (specifically, 16). We now shift the focus of our discussion to these critical optimal load factors.

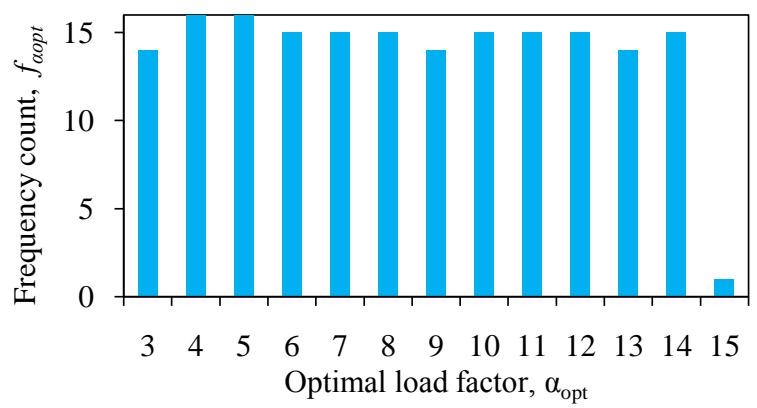

Figure2:The frequency distributions of the optimal load factors

Table 1 summarizes the corresponding optimal parameters obtained from Equations (6) through (9) for these critical optimal load factors obtained in Figure 2.

Table 1. Values of the optimal parameters corresponding to the critical optimal load factors

\begin{tabular}{|c|c|c|c|c|}
\hline $\boldsymbol{\alpha}_{\text {optMax }}$ & $\boldsymbol{c}_{\text {opt }}$ & $\boldsymbol{P}_{\text {lopt }}$ & $\boldsymbol{P}_{\text {2opt }}$ & $\boldsymbol{\rho}_{\text {oopt }}$ \\
\hline 4 & 2.50 & 0.8005 & 0.5304 & 0.3508 \\
\hline 5 & 3.30 & 0.8404 & 0.5108 & 0.2588 \\
\hline
\end{tabular}

In what follows, we examine the effect of $\rho_{\text {aopt }}$ on the choice of the number of independent projections, $k$; the number of tables, $L$; the computational complexity to create the data structure; the memory requirementfor the data structure;and the query runtime.For simplicity, we use $\alpha, c, P_{1}, P_{2}$, and $\rho$ to mean $\alpha_{\text {optMax }}, c_{o p t}, P_{\text {lopt }}, P_{2 o p t}$, and $\rho_{\text {oopt }}$ respectively.

\subsection{Choosing Parameters $k$ and $L$}

From Equation(2), the probability of collision for a single scalar projection is $P_{1}$. Let $\delta$ be the probability of false negatives, i.e. the probability of failure to return a true nearest neighbor as an output to a given query data point. A typical choice of $\delta$ is $0.1[30]$.Now, for $k_{l}$ independent projections the probability of collision becomes $P_{1}^{k}$ thus, making the ratio $P_{1} / P_{2}$ larger. Consequently, the probability of no collision under all $k$ projections is given by $1-P_{1}^{k}$. To further increase the chance of close data points hashing to the same bucket with a high probability, a family of these hash functions is chosen independently for a number of $L$ tables. This means that the probability of no collision under all $L_{I}$ tablesis $\left(1-P_{1}^{k}\right)^{L}$. Requiring that this probability is bounded below by $\delta$ i.e. $\left(1-P_{1}^{k}\right)^{L} \geq \delta$, yields Equation(10).

$$
L \geq \frac{\ln \delta}{\ln \left(1-P_{1}^{k}\right)}
$$

This becomes a one-degree of freedom design. Thus, the remaining parameter, $k$ is chosen using Johnson-Lindenstrauss Lemma as shown in Equation(11). Once $k$ is known, $L$ can be computed.

$$
k=\frac{\ln n}{\ln \left(1 / P_{2}\right)}
$$

For a fixed value of $P_{2}, k$ increases monotonically with $n$ and $L$ increases monotonically with $k$ respectively. The $L$ can be expressed in terms of $\rho$ as shown in Equation (12). 


$$
L \geq \frac{\ln \delta}{\ln \left(1-P_{1}^{k}\right)}
$$

To make the computational complexity and memory analyses easier, the lower bound of $L$ is approximated using Newton's Binomial Theorem as shown in Equation (13).

$$
L \approx n^{\rho} \ln (1 / \delta)
$$

Figure 3 and Figure4show variations of $k$ and $L$ with $n$ respectively.

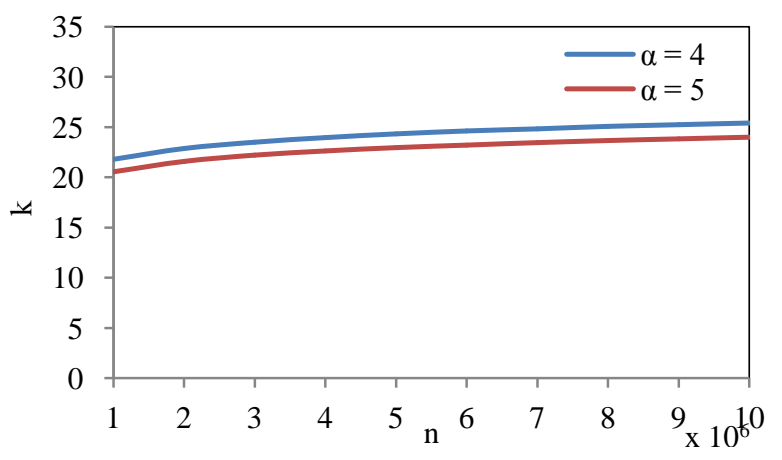

Figure3:Variation of $k$ with $n$ for $\alpha=4$ and $\alpha=5$

From Figure3, it should be noted that the value of $k$ is not significantly affected by the choice of $\alpha=4$ or $\alpha=5$.

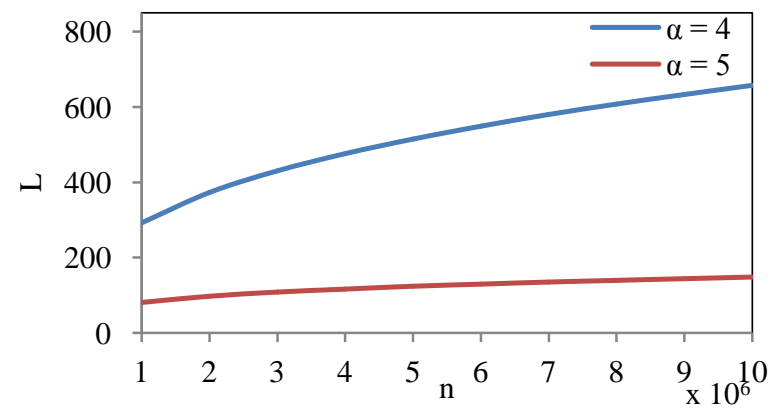

Figure4:Variation of $L$ with $n$ for $\alpha=4$ and $\alpha=5$

\section{Complexity Analyses}

The main goal of selecting optimal parameters for LSH is to enable fast computations of the hash values; and to use as little memory space as possible to store the computed hash values. These are necessary in order to guarantee fast query runtime. In what follows we analyze the computational complexity, the memory requirement and the query runtime for LSH in general and more specifically for the load factors $\alpha=4$ (mostly used) and $\alpha=5$.

\subsection{Computational Complexity}

This is the total number of computations required to compute the hash values for the $n$ data points using $k$ hash functions to create $L$ tables. Suppose $h_{i j}$ is the $\mathrm{i}^{\text {th }}$ hash function for the $\mathrm{j}^{\text {th }}$ table. Assuming that it takes one computational operation to project a data point $\boldsymbol{p}$ in the direction of $h_{\mathrm{ij}}\left(\right.$ i.e. $\left.\boldsymbol{h}_{\boldsymbol{i} \boldsymbol{j}} \bullet \boldsymbol{p}\right)$. To make $k$ projections, requires $k$ operations. To project $p$ onto all the $L$ tables requires $k L$ operations. Thus, to project all $n$ data points requires $n k L$ operations. As a result, the computational complexityis $O(n k L)$. The hidden constant in
$O(n k L)$ depends on the dimensionality of $\boldsymbol{p}$. For a fixed $n$, the computational complexity grows as a function of $k$ and $L$.This implies that both $k$ and $L$ must be at their best minimum in order to ensure low computational complexity. From Equation(10), $k$ monotonically decreases as $P_{2}$ decreases. To ensure that $k$ is small, $P_{2}$ must be chosen as small as possible. From Equation(11), $L$ decreases with decreasing $k$. From Equation(4), $\rho$ decreases as $P_{1}$ increases and $P_{2}$ decreases. Thus, $P_{1}$ must be chosen as large as possible.Table 2 shows the computational complexitiesfor using the existing optimal load factor used by researchers over a decade; and using our proposed optimal load factor.

Table 2.Computational complexities for $\alpha=4$ and $\alpha=5$

\begin{tabular}{|c|c|}
\hline $\boldsymbol{\alpha}$ & Computational Complexity \\
\hline 4 & $O\left(n^{1.3508} \ln (1 / \delta) \ln n\right)$ \\
\hline 5 & $O\left(n^{1.2588} \ln (1 / \delta) \ln n\right)$ \\
\hline
\end{tabular}

The computational complexities grow logarithmically as $n$ increasesfor $\alpha=4$ and $\alpha=5$ as shown in Figure5.

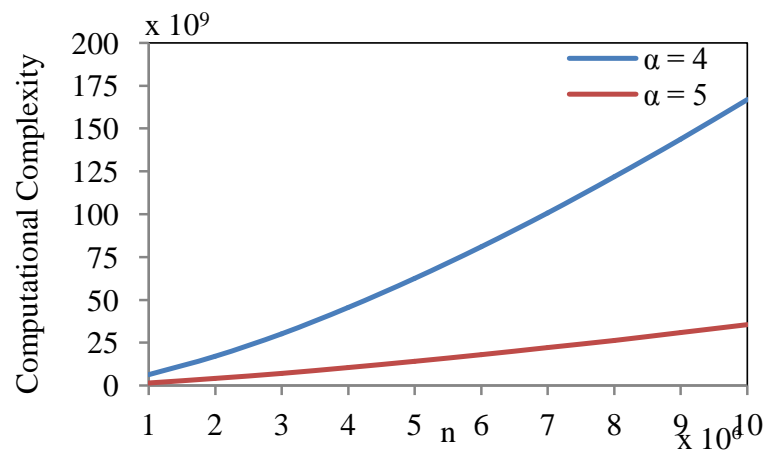

Figure5:Thecomputational complexityfor $\alpha=4$ and $\alpha=5$

The unit for the computational complexity is number of operations. In an actual implementation of the hash tables, this is expressed in seconds. The computational complexity saving denoted CCS, for using $\alpha=5$ over $\alpha=4$ can be approximatedbyEquation (14).

$$
\mathrm{CCS} \approx\left(1-\frac{1}{n^{0.1}}\right) * 100 \%
$$

Figure6 shows the computational complexity saving of the proposed optimal load factor over the existing one.

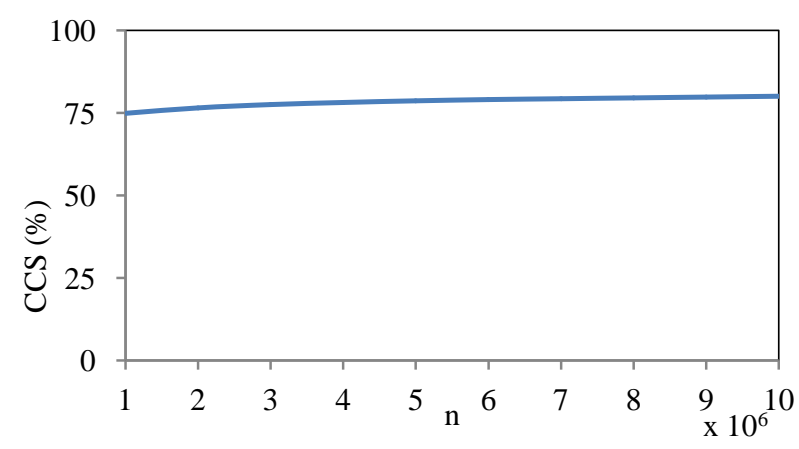

Figure6: Computational complexity saving for $\alpha=5$ over $\alpha=4$

From Figure6, for just a million data points (i.e. $n=10^{6}$ ) 
there is approximately $75 \%$ less computations for using $\alpha=5$ compared to using $\alpha=4$.

\subsection{Memory Requirement}

This is the memory space required to store the $n$ data points themselves along with their respective hash values. To store each data point (i.e. $\boldsymbol{p} \in \mathfrak{R}^{d}$ ) along with its $k$ concatenated hash values on all $L$ tables requires $L(d+k)$ memory. If the $k$-concatenated values are hashed to produce a single integer, then the memory requirement reduces to $L(d+1)$ per data point. For all $n$ data points, the memory requirement becomes $O(d n L+n L)$. For a large data set this can be huge. In our implementation however, two optimization techniques are used to reduce the memory requirement further. First, all the $n$ data points in $\mathcal{P}$ are indexed from 1 through $n$. The indexing is the same across all the $L$ hash tables. This means that each data point is stored once instead of $L$ times. This reduces the memory to $O(d n+n L)$. Second, the single integer produced from hashing the $k$-concatenated values is not stored but rather used to point to a bucket. The index of the data point is then stored in that bucket. Thus, each data point can be referred to by an index. The hidden constant in $O(d n)$ depends on the data type of the data points in the dataset. Henceforth, the memory analysis concentrates on the hash tables of the data structure. Thus the memory requirement to store the hash tables for all the $n$ data point becomes $O(n L)$. Once again, $L$ has to be kept at its best minimum to achieve the best memory space. The hidden constant in $O(n L)$ is the number of bytes required to point to each bucket and $0.125\left\lceil\log _{2} n\right\rceil$ bytes to store each index of the data point on each table. For example, for a billion data points (i.e. $n \approx 2^{30}$ ) the hidden constant becomes eight bytes. Table 3 shows the memory requirement for using the existing optimal load factor and our proposed optimal load factor.

Table 3. Memory requirements for $\alpha=4$ and $\alpha=5$

\begin{tabular}{|c|c|}
\hline $\boldsymbol{\alpha}$ & Memory Requirement \\
\hline 4 & $O\left(n^{1.3508} \ln (1 / \delta)\right)$ \\
\hline 5 & $O\left(n^{1.2588} \ln (1 / \delta)\right)$ \\
\hline
\end{tabular}

The memory requirementsgrow with $n$. No graph is provided since the expressions on Table 3 are similar to those obtained in Table 2. It is however worth mentioning that he memory saving denoted as MS, for using $\alpha=5$ over $\alpha=4$ can be approximatedby Equation (15).

$$
\mathrm{MS} \approx\left(1-\frac{1}{\mathrm{n}^{0.1}}\right) * 100 \%
$$

From Equation (15), for just a million data points (i.e. $n=10^{6}$ ) the memory saving for using $\alpha=5$ instead of $\alpha=$ 4isapproximately $75 \%$ and this increases as $n$ increases.

\subsection{Query Runtime}

When running a query $\boldsymbol{q}$, two time complexities are involved. First, the time required to hash the query to a bucket on each of the $L$ tables to retrieve the candidate set. Second, the time required to compute the distance between the query and the entries in the candidate set. Let $\tau_{h}$ and $\tau_{c}$ denote these respectively. Computing the hash value $h_{j}(\widehat{\boldsymbol{p}})$ for all the tables is $O(k L)$. The second level hash value computation is $O(L)$ and it is relatively insignificant compared to that of the first. Thus, $\tau_{h}$ is $O(k L)$. Suppose it takes one computational operation to compute the $l_{2}$ norm between $\boldsymbol{q}$ and an entry in the candidate set. The expected number of entries in the candidate set is $\alpha L$. Thus, $\tau_{c}$ becomes $O(\alpha L)$. As a result, the total query runtime is $O(k L+\alpha L)$.

If the $(R, c)$-NNs are to be sorted then, the computed $l_{2}$ norms have to be sorted using a sorting algorithm such as a quick sort. Let this be denoted by $\tau_{s}$. The quick sort average computational complexityis $O\left(\alpha L \log _{2} \alpha L\right)$. Thus, the total query runtime becomes $\tau_{h}+\tau_{c}+\tau_{s}$. The hidden constants in these analyses depend on the dimensionality and the complexity of the data points.

Neglecting the complexities due to computing the distances between the query point and the items in the retrieved buckets and neglecting the complexity due to sorting these distances, the query time complexity is dependent only on the table lookup. Thus, the query time complexity becomes $O\left(n^{\rho} \ln (1 /\right.$ $\delta) \ln n$ ) with a hidden constant factor of $-\rho / \ln P_{1}$ (usually less than 2).The theoretical query runtime gain, $G$ for using $\alpha=5$ over $\alpha=4$ canthen be approximated by Equation(16).

$$
G \approx n^{0.1}
$$

The $G$, is the ratio of the query runtime for using $\alpha=5$ to that of using $\alpha=4$. From Equation (16),for just a million data points (i.e. $n=10^{6}$ ), the query runtime gain for $\alpha=5$ is approximatelyfour times relative to $\alpha=4$.

\section{PRACTICALLSH IMPLEMENTATION}

We present a real-world problem and solve it using LSH parameterization based on existing optimal load factor of $\alpha=4$ and our proposed optimal load factor, $\alpha=5$. It must be emphatically stated that the performance of the LSH algorithm is not dependent on the dataset used. This was evident in all the comparative analyses thathave been conducted in the previous sections. This experiment is to onlyprovide a sample test of the LSH under the proposed load factor compared to the existing load factor. Consequently, there is no need to test the new parameterization of the LSH with several datasets in order to justify that LSH using the proposed load factor always outperforms that of the existing load factor.

\subsection{Dataset}

The algorithm is tested on real texture features extracted from Defense Meteorological Satellite Program (DMSP) satellite images. The DMSP began in 1991. The visible and infrared sensors collect images across a $3000 \mathrm{~km}$ swath, providing global coverage twice per day. Currently, the National Geophysical Data Center (NGDC) receives and processes approximately $8.5 \mathrm{~GB}$ of satellite imagery data per day from four DMSP satellites. Each image is downsized to363 $\times 293$. The texture featuresare extracted for 1.6 million images. The dimension for each texture feature vector is $1 \times 10$ (i.e. $d=10$ ) and consists floating point numbers. These features are based on normalized central moments of wavelet edges after multi-resolution decomposition of each image. A detailed discussion of the texture feature extraction approach could be found in[42].

The LSH parameterization we formulate is to help quickly find the similar images to a given query image based on Euclidean metric space. The similar images may assist scientists, meteorologists, and data analysts to integrate these 
into their scientific predictive models to make real-time predictions such as when and where a hurricane may strike.

\subsection{Parameterization}

Table 4 lists the input parameters and their computed values required to build the hash tables for the dataset using the traditional optimal load factor of $\alpha=4$ and our proposed optimal load factor of $\alpha=5$.

Table 4 Corresponding inputs to existing load factor and proposed load factor for $n=1.6 \times 10^{6}$

\begin{tabular}{|c|c|c|c|c|c|c|}
\hline Inputs & $\boldsymbol{\alpha}$ & $\boldsymbol{c}$ & $\boldsymbol{\rho}$ & $\boldsymbol{m}$ & $\boldsymbol{k}$ & $\boldsymbol{L}$ \\
\hline Existing & 4 & 2.5 & 0.3508 & 400009 & 23 & 383 \\
\hline Proposed & 5 & 3.3 & 0.2588 & 320009 & 22 & 105 \\
\hline
\end{tabular}

On Table $4, m$ is the size of each hash table. That is the number of buckets on each table and this is computed as a prime approximation of $\lceil n / \alpha\rceil$. The ceiling operator $\lceil\bullet\rceil$ must be applied to both $k$ and $L$ since both have to be positive integers $\left(k, L \in Z^{+}\right)$.

\subsection{Hash Table Creation}

Below are the steps for building the hash tables for the $n$ data points in the dataset. The creation of the hash tables takes time. As a result, they are created only once and stored along with their families of hash functions. The tables are then used to answer different queries in sublinearqueryruntime.

(1) Generate $L$ families of hash functions, $h_{1}, h_{2}, \ldots, h_{L}$ randomly and independently from $\mathcal{N}(0,1)$ such that each $h_{j}=\left[\boldsymbol{h}_{\mathbf{1 j}}, \boldsymbol{h}_{\mathbf{2 j}}, \ldots, \boldsymbol{h}_{\boldsymbol{k} j}\right]$ and each hash function, $\boldsymbol{h}_{\boldsymbol{i j}} \in \mathfrak{R}^{d} \forall j \in[1,2, \ldots, L]$ and $\forall i \in[1,2, \ldots, k]$

(2) Generate an offset, $b_{i j}$ randomly, independently and uniformly from $[0, \alpha]$ for each $\mathrm{i}^{\text {th }}$ hash function in each $\mathrm{j}^{\text {th }}$ family

(3) Generate $L$ set, $H_{1}, H_{2}, \ldots, H_{L}$ of random integers from the range [1 $\mathrm{m}$ ], independently such that each $H_{j} \in \mathfrak{R}^{k}$

(4) Index all the $n$ data points in $\mathcal{P}$ from 1 through $n$

(5) For each feature vector $\boldsymbol{p} \in \mathcal{P}$, normalized $\boldsymbol{p}$ as $\widehat{\boldsymbol{p}}=\boldsymbol{p} /\|\boldsymbol{p}\|_{2}$

(6) Compute the hash value for $\widehat{\boldsymbol{p}}$ for the $\mathrm{j}^{\text {th }}$ family as $h_{j}(\hat{\boldsymbol{p}})=\left[\left\lfloor\frac{\boldsymbol{h}_{\mathbf{1}} \cdot \hat{\boldsymbol{p}}+b_{1 j}}{\alpha}\right\rfloor,\left\lfloor\frac{\boldsymbol{h}_{2 j} \boldsymbol{\bullet} \widehat{\boldsymbol{p}}+b_{2 j}}{\alpha}\right\rfloor, \ldots,\left\lfloor\frac{\boldsymbol{h}_{\boldsymbol{k} \boldsymbol{}} \cdot \hat{\boldsymbol{p}}+b_{k j}}{\alpha}\right\rfloor\right]$

(7) Compute the second level hash value for the $\widehat{\boldsymbol{p}}$ as $h_{j}^{*}(\widehat{\boldsymbol{p}})=\left(\left(h_{j}(\widehat{\boldsymbol{p}}) \cdot H_{j}\right) \bmod M\right) \bmod m$

(8) Store the index of $\boldsymbol{p}$ in the bucket $h_{j}^{*}(\widehat{\boldsymbol{p}})$ on the $\mathrm{j}^{\text {th }}$ table

The $M$ is a large prime integer close to $2^{W}$ where, $W$ is the word width of the microprocessor being used. For a 64-bit computer, $M=2^{64}-5$. In [43, 44],we offered more details regarding the formulation of the equation for computing the hash values.

\subsection{Bucket Hashing}

Below are the steps to find the $(R, c)$-NNs to a query point.

(1) For a given query feature vector, $\boldsymbol{q} \in \mathcal{P}$ normalized $\boldsymbol{q}\left(\right.$ i.e. $\left.\widehat{\boldsymbol{q}}=\boldsymbol{q} /\|\boldsymbol{q}\|_{2}\right)$

(2) Compute the hash values for $\widehat{\boldsymbol{q}}$ as $h_{1}(\widehat{\boldsymbol{q}}), h_{2}(\widehat{\boldsymbol{q}}), \ldots, h_{L}(\widehat{\boldsymbol{q}})$
(3) Compute the second level hash values as $h_{1}^{*}(\widehat{\boldsymbol{q}}), h_{2}^{*}(\widehat{\boldsymbol{q}}), \ldots, h_{L}^{*}(\widehat{\boldsymbol{q}})$

(4) Use the indices in these buckets to collect their corresponding feature vectors (let us call this the candidate set)

(5) Compute the $l_{2}$ norm between $\boldsymbol{q}$ and the entries in the candidate set

(6) Retrieve the top $K,(R, c)$-NNs to $\boldsymbol{q}$ or terminate the search once $2 L$ (including duplicates) items are retrieved

In our implementation, the number of nearest neighbors to be found closest to $\boldsymbol{q}$ is $K=50$ (must be less than $2 L$ ). The choice of $R$ is better controlled if the data points are normalized. In the $l_{2}$ normalized space, the $l_{2}$ norm between any two data points, $\widehat{\boldsymbol{p}}$ and $\widehat{\boldsymbol{q}}$ can be computed using Equation (17)

$$
\|\widehat{\boldsymbol{p}}-\widehat{\boldsymbol{q}}\|_{2}=\sqrt{2(1-\hat{\boldsymbol{p}} \cdot \widehat{\boldsymbol{q}})}
$$

From Equation (17), $0 \leq\|\hat{\boldsymbol{p}}-\widehat{\boldsymbol{q}}\|_{2} \leq 2$. Let $\lambda$ be the fraction of the maximum distance within which each $(R, c)$-NNs must lie. This means that $c R$ is bounded below by $2 \lambda$. Consequently, the upper bound of $R$ can be computed as in Equation (18) for any approximation factor, $c$.

$$
R=\frac{2 \lambda}{c}
$$

It should be noted that if $\lambda$ is large, the search domain becomes bigger. On the other hand, if $\lambda$ is too small, the search domain becomes small and only few nearest neighbors could be found. For a highly sparse dataset, $\lambda$ has to be kept relatively high while for highly dense dataset, $\lambda$ has to be kept relatively small. We keep $\lambda$ at $5 \%$ (retrieved data points are within 95 percentile of the maximum distance). Thus $R$ becomes 0.0303 units.

\subsection{Result and Discussion}

Two sets of hash tables are createdusing a 64-bit Intel (R) core (TM) 880 i7 CPU at $3.07 / 3.20 \mathrm{GHz}$ with $16 \mathrm{~GB}$ RAM. The firstset has 383 hash tables, each having 400009 buckets. This set corresponds to $\alpha=4$. The second set which corresponds to $\alpha=5$, has 105 hash tables, each having 320009 buckets. The two sets of hash tables are stored together with their respective families of hash functions. Table 5 summarizes the computational complexities and the memory requirements of the two sets of hash tables.

Table 5 The computational complexities (CC) and memory requirements (MR) for $\alpha=4$ and $\alpha=5$

\begin{tabular}{|c|c|c|c|c|}
\hline$\alpha$ & CC (s) & CCS (\%) & $\begin{array}{c}\text { MR } \\
(\text { MB) }\end{array}$ & MS (\%) \\
\hline 4 & 13676 & - & 3092 & - \\
\hline 5 & 3740 & 73 & 606 & 80 \\
\hline
\end{tabular}

From Table 5, under the proposed load factor of $\alpha=5$, the hash tables are created in much smaller time compared to the time taken to create the hash tables under the existing load factor of $\alpha=4$.In other words, there is $73 \%$ less computations to create the hash tables using $\alpha=5$ compared to using $\alpha=$ 4.This is due to the fact our proposed technique creates fewer hash tables that are just sufficient enough to report the NNs 
correctly. In fact, our proposed choice of $\alpha=5$ reduces the $L$ to more than a third compared to that of $\alpha=4$ (see Table 4). In addition, the memory required to store the hash tables is much smaller under the proposed load factor. To store the hash tables, approximately $80 \%$ memory space is saved for using $\alpha=5$ compared to using $\alpha=4$.The theoretical CCS and MS are approximately $75 \%$ each (see Equations 15 and 16).

Once the tables are created and stored, they can be used to answer queries. Since the data points are indexed, a query is simply referred to by its index. The two sets of hash tables are presented with same ten queries chosen randomly from the dataset and the goal is to report the top $50(R, c)$-NNs to each. Each query is run through a Linear Search (LS) to find the top 50 exact NNs ('gold standard'). Two comparisons are made. First, the $50(R, c)$-NNs for each query reported by each choice for $\alpha$ are compared to that of the LS to compute the retrieval percentage accuracy. Second, the query runtimes ( $\tau_{L S H 4}$ and $\tau_{L S H 5}$ for $\alpha=4$ and $\alpha=5$ respectively) are compared to the query runtime $\left(\tau_{L S}\right)$ of the LS.These ratios are denoted as $\mathrm{G}_{4}$ and $\mathrm{G}_{5}$ (gains). $G_{4}=\tau_{L S H_{4}} / \tau_{L S}$ and $G_{5}=$ $\tau_{L S H 5} / \tau_{L S}$ for $\alpha=4$ and $\alpha=5$ respectively. The query runtime gain of using $\alpha=5$ over $\alpha=4$ is given by $G=$ $\tau_{L S H} / \tau_{L S H 5}$. The sizes of the candidate sets are expressed as fraction of the number of data points, $n$ and are denoted by $\mathrm{CSs}_{4}$ and $\mathrm{CSs}_{5}$ respectively.

Table6 summarizes these results for the ten queries, which are represented by their indices.

For visual purposes, Figure 7 and Figure 8 show one sample query image (the $970997^{\text {th }}$ image) and its top five similar visual and thermal images respectively. The images are ranked; ' 1 ' being the best similar image and ' 5 ' being the worst similar image found respectively.
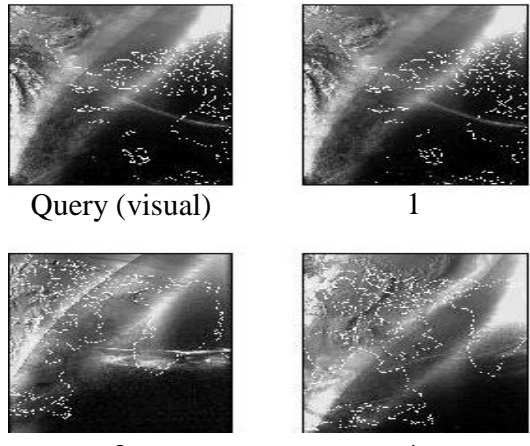

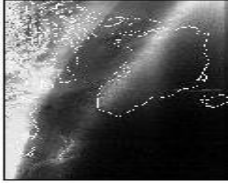

2

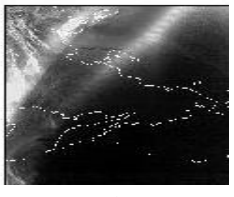

5

Figure 7: Visual query image and its top five matches

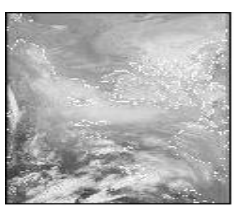

Query (thermal)

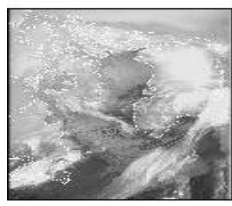

3

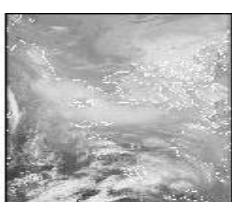

1

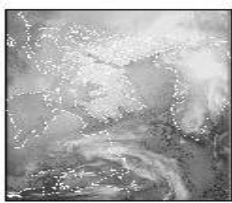

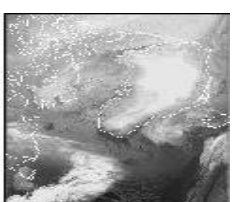

2

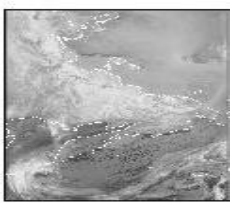

Figure 8: Thermal query image and its top five matches

Table6.Summarized results for ten random queries reference by their indices

\begin{tabular}{|c|c|c|c|c|c|c|c|c|c|c|}
\hline \multirow{2}{*}{$\begin{array}{l}\text { Query } \\
\text { index }\end{array}$} & \multicolumn{2}{|c|}{ Retrieval Accuracy (\%) } & \multicolumn{3}{|c|}{ Query Runtime (ms) } & \multicolumn{3}{|c|}{ Gain } & \multicolumn{2}{|c|}{ Candidate Set size (\%) } \\
\hline & $\alpha=4$ & $\alpha=5$ & $\tau_{L S}$ & $\tau_{L S H 4}$ & $\tau_{\text {LSH5 }}$ & $\mathrm{G}_{4}$ & $\mathrm{G}_{5}$ & $\mathrm{G}$ & $\mathrm{CSs}_{4}$ & $\mathrm{CSs}_{5}$ \\
\hline 412014 & 100 & 100 & 2824.30 & 52.87 & 27.10 & 53 & 104 & 2.0 & 0.28 & 0.41 \\
\hline 497945 & 100 & 100 & 2600.94 & 26.88 & 12.69 & 97 & 205 & 2.1 & 0.28 & 0.25 \\
\hline 783624 & 100 & 100 & 2678.10 & 35.34 & 12.17 & 76 & 220 & 2.9 & 0.28 & 0.28 \\
\hline 970997 & 100 & 100 & 2558.26 & 37.87 & 10.82 & 68 & 236 & 3.5 & 0.27 & 0.23 \\
\hline 1011775 & 100 & 100 & 2677.33 & 20.16 & 11.35 & 133 & 236 & 1.8 & 0.18 & 0.22 \\
\hline 1084917 & 100 & 100 & 2767.25 & 26.04 & 16.33 & 106 & 169 & 1.6 & 0.35 & 0.38 \\
\hline 1191509 & 100 & 100 & 2727.99 & 19.52 & 12.29 & 140 & 222 & 1.6 & 0.19 & 0.27 \\
\hline 1211521 & 100 & 100 & 2653.87 & 27.85 & 16.36 & 95 & 162 & 1.7 & 0.32 & 0.4 \\
\hline 1285384 & 100 & 100 & 3121.96 & 27.99 & 20.54 & 112 & 152 & 1.4 & 0.33 & 0.57 \\
\hline 1507281 & 100 & 100 & 2621.44 & 23.07 & 14.55 & 114 & 180 & 1.6 & 0.25 & 0.36 \\
\hline Average & 100 & 100 & 2723.14 & 29.76 & 15.42 & 99 & 189 & 2.1 & 0.27 & 0.34 \\
\hline
\end{tabular}

From Table 6 the average query runtime gain of using $\alpha=5$ over using $\alpha=4$ is approximately 2 .It should be noted that both schemes reported the top $50 \mathrm{NNs}$ correctly compared to those reported by the LS. These similar images are same as those reported by the LS.

For the results shown in Figure 7 and Figure 8, the LSH scheme corresponding to $\alpha=5$ searched only $\mathbf{0 . 2 3 \%}$ whiles that corresponding to $\alpha=4$ searched $\mathbf{0 . 2 7 \%}$ of the dataset. Usually we expect $\mathrm{CSS}_{5}<\mathrm{CSS}_{4}$ but this is not necessarily the case because the candidate set is the union of the entries in all the buckets collected. If the union operator is not applied then indeed $\mathrm{CSs}_{5}$ would always be less than $\mathrm{CSs}_{4}$.

\section{CONCLUSION}

In a large dataset retrieval application in which an approximate match is as good and acceptable as an exact match, LSH is very effective. Unlike the LS, hash tables need to be created when using LSH and this takes time. But once this is done and stored,the benefit of the LSH outweighs that of LS in terms of the query runtime complexity. The goal of the LSH is to search a fraction of the dataset to find the $(R, c)$-NNs for any given query data point.This makes LSH scalable for searching large dataset. The number of tables created and the number of projections used have a significant effect on the performance of the LSH. We have shown both theoretically and practically that for $\alpha=5$, the LSH achieves lower computational complexity, lower memory requirement and faster query runtime than usingthe traditional 
optimal load factor of $\alpha=4$. We therefore propose the use of $\alpha=5$ as an optimal load factor under the LSH scheme.

The parameterization discussed based on the $l_{2}$ norm is extendable to all fractional norms as well. In general, LSH is not effective for small dataset.

\section{ACKNOWLEDGEMENT}

This work is partly supported by the Expeditions in Computing by the National Science Foundation (NSF) under Award CCF1029731 and by National Oceanic and Atmospheric Administration/National Geophysical Data Center (NOAA/NGDC) Educational Program under Cooperative Agreement No: NA060AR4810187. We are very grateful to NSF and NOAA/NGDC

\section{REFERENCES}

[1] Arya, S., Mount, D. M., Netanyahu, N. S., Silverman, R., and Wu, A. Y. 1998. "An optimal algorithm for approximate nearest neighbor searching fixed dimensions," J. ACM, vol. 45, no. 6, pp. 891-923, 1998).

[2] Flickner, M., Sawhney, H., Niblack, W., Ashley, J., Qian, H., Dom, B., Gorkani, M., Hafner, J., Lee, D., Petkovic, D., Steele, D., and Yanker, P. 1995. "Query by image and video content: the QBIC system," Computer, vol. 28, no. 9, pp. 23-32, 1995).

[3] Fayyad, U. M. 1996. Advances in knowledge discovery and data mining: AAAI Press.

[4] Lin, K. I., Jagadish, H. V., and Faloutsos, C. 1994. "The TV-tree: an index structure for high-dimensional data," The VLDB Journal, vol. 3, no. 4, pp. 517-542, 1994).

[5] Roussopoulos, N., Kelley, S., and Vincent, F. 1995 "Nearest neighbor queries," in Proceedings of the 1995 ACM SIGMOD international conference on Management of data, San Jose, California, United States, pp. 71-79.

[6] White, D. A., and Jain, R. "Similarity indexing with the SS tree," Data Engineering, 1996. Proceedings of the Twelfth International Conference on. pp. 516-523.

[7] Berchtold, S., Keim, D. A., and Kriegel, H.-P. 1996. "The X-tree: An Index Structure for High-Dimensional Data," in Proceedings of the 22th International Conference on Very Large Data Bases, pp. 28-39.

[8] Berchtold, S., Böhm, C., Keim, D. A., and Kriegel, H.-P. 1997. "A cost model for nearest neighbor search in highdimensional data space," in Proceedings of the sixteenth ACM SIGACT-SIGMOD-SIGART symposium on Principles of database systems, Tucson, Arizona, United States, pp. 78-86.

[9] Cleary, J. G. 1979. "Analysis of an Algorithm for Finding Nearest Neighbors in Euclidean Space," ACM Trans. Math. Softw., vol. 5, no. 2, pp. 183-192, 1979).

[10] Bentley, J. L., Weide, B. W., and Yao, A. C. 1980. "Optimal Expected-Time Algorithms for Closest Point Problems," ACM Trans. Math. Softw., vol. 6, no. 4, pp. 563-580, 1980).

[11] Friedman, J. H., Bentley, J. L., and Finkel, R. A. 1977. “An Algorithm for Finding Best Matches in Logarithmic Expected Time," ACM Trans. Math. Softw., vol. 3, no. 3, pp. 209-226, 1977).

[12] Sproull, R. 1991. "Refinements to nearest-neighbor searching in k-dimensional trees," Algorithmica, vol. 6, no.
1, pp. 579-589, 1991).

[13] Arya, S., and Mount, D. M. 1995. "Approximate range searching," in Proceedings of the eleventh annual symposium on Computational geometry, Vancouver, British Columbia, Canada, pp. 172-181.

[14] Preparata, F. P., and Shamos, M. I. 1985. Computational Geometry: An Introduction: Springer-Verlag.

[15] Edelsbrunner, H. 2004. Algorithms in Combinatorial Geometry: Springer.

[16] de Berg, M., Cheong, O., van Kreveld, M., and Overmars, M. 2008. Computational Geometry: Algorithms and Applications: Springer.

[17] Yao, A. C., and Yao, F. F. 1985. "A general approach to ddimensional geometric queries," in Proceedings of the seventeenth annual ACM symposium on Theory of computing, Providence, Rhode Island, United States, pp. 163-168.

[18] Clarkson, K. L. 1988. "A randomized algorithm for closestpoint queries," SIAM J. Comput., vol. 17, no. 4, pp. 830847, 1988).

[19] Agarwal, P. K., and Matoušek, J. 1993. "Ray shooting and parametric search," SIAM J. Comput., vol. 22, no. 4, pp. 794-806, 1993).

[20] Meiser, S. 1993. "Point location in arrangements of hyperplanes," Inf. Comput., vol. 106, no. 2, pp. 286-303, 1993).

[21] Gionis, A., Indyk, P., and Motwani, R. 1999. "Similarity Search in High Dimensions via Hashing," in Proceedings of the 25th International Conference on Very Large Data Bases, pp. 518-529.

[22] Weber, R., Schek, H. J., and Blott, S. 1998. "A Quantitative Analysis and Performance Study for Similarity-Search Methods in High-Dimensional Spaces," in Proceedings of the 24rd International Conference on Very Large Data Bases, pp. 194-205.

[23] Arya, S., Mount, D. M., Netanyahu, N. S., Silverman, R., and Wu, A. 1994. "An optimal algorithm for approximate nearest neighbor searching," in Proceedings of the fifth annual ACM-SIAM symposium on Discrete algorithms, Arlington, Virginia, United States, pp. 573-582.

[24] Har-Peled, S. "A Replacement for Voronoi Diagrams of Near Linear Size," 42nd IEEE symposium on Foundations of Computer Science. pp. 94-94.

[25] Indyk, P., and Motwani, R. 1998. "Approximate nearest neighbors: towards removing the curse of dimensionality," in Proceedings of the thirtieth annual ACM symposium on Theory of computing, Dallas, Texas, United States, pp. 604-613.

[26] Kleinberg, J. M. 1997. "Two algorithms for nearestneighbor search in high dimensions," in Proceedings of the twenty-ninth annual ACM symposium on Theory of computing, El Paso, Texas, United States, pp. 599-608.

[27] Kushilevitz, E., Ostrovsky, R., and Rabani, Y. 1998 "Efficient search for approximate nearest neighbor in high dimensional spaces," in Proceedings of the thirtieth annual ACM symposium on Theory of computing, Dallas, Texas, United States, pp. 614-623.

[28] Beyer, K. S., Goldstein, J., Ramakrishnan, R., and Shaft, U. 
1999. "When Is "Nearest Neighbor" Meaningful?," in Proceedings of the 7th International Conference on Database Theory, pp. 217-235.

[29] Hinneburg, A., Aggarwal, C. C., and Keim, D. A. 2000. "What Is the Nearest Neighbor in High Dimensional Spaces?," in Proceedings of the 26th International Conference on Very Large Data Bases, pp. 506-515.

[30] Datar, M., Immorlica, N., Indyk, P., and Mirrokni, V. S. 2004. "Locality-sensitive hashing scheme based on p-stable distributions," in Proceedings of the twentieth annual symposium on Computational geometry, Brooklyn, New York, USA, pp. 253-262.

[31] Andoni, A., and Indyk, P. 2006. "Near-Optimal Hashing Algorithms for Approximate Nearest Neighbor in High Dimensions," in Proceedings of the 47th Annual IEEE Symposium on Foundations of Computer Science, pp. 459. 468

[32] Slaney, M., and Casey, M. 2008. "Locality-Sensitive Hashing for Finding Nearest Neighbors [Lecture Notes]," Signal Processing Magazine, IEEE, vol. 25, no. 2, pp. 128 131, 2008).

[33] Cormen, T. H., Leiserson, C. E., Rivest, R. L., and Stein, C. 2001. Introduction to Algorithms, Second Edition, p.^pp. 224 -252: MIT Press.

[34] Nolan, J. 2007. Stable Distributions: Models for HeavyTailed Data: Springer Verlag.

[35] Buhler, J. 2001. "Efficient large-scale sequence comparison by locality-sensitive hashing," Bioinformatics, vol. 17, no. 5, pp. 419-428, 2001).

[36] Buhler, J. 2002. "Provably sensitive Indexing strategies for biosequence similarity search," in Proceedings of the sixth annual international conference on Computational biology, Washington, DC, USA, pp. 90-99.
[37] Buhler, J., and Tompa, M. 2002. "Finding motifs using random projections," J Comput Biol, vol. 9, no. 2, pp. 225242, 2002)

[38] Ouyang, Z., Memon, N. D., Suel, T., and Trendafilov, D. 2002. "Cluster-Based Delta Compression of a Collection of Files," in Proceedings of the 3rd International Conference on Web Information Systems Engineering, pp. 257-268.

[39] Shivakumar, N. 1999. "Detecting digital copyright violations on the internet," Stanford University

[40] Cheng, Y. "MACS: music audio characteristic sequence indexing for similarity retrieval," Applications of Signal Processing to Audio and Acoustics, 2001 IEEE Workshop on the. pp. 123-126.

[41] Zolotarev, V. M. $1986 . \quad$ One-Dimensional Stable Distributions: American Mathematical Society.

[42] Gebril, M., Buaba, R., Homaifar, A., and Kihn, E. "Structural indexing of satellite images using automatic classification," Aerospace Conference, 2011 IEEE. pp. 1-7.

[43] Buaba, R., Homaifar, A., Gebril, M., and Kihn, E. "Satellite image retrieval application using Locality Sensitive Hashing in $\mathrm{L}_{2}$-space," Aerospace Conference, 2011 IEEE. pp. 1-7.

[44] Buaba, R., Homaifar, A., Gebril, M., Kihn, E., and Zhizhin, M. 2011. "Satellite image retrieval using low memory locality sensitive hashing in Euclidean space," Earth Science Informatics, vol. 4, no. 1, pp. 17-28, (2011/03/01 2011). 